\title{
Maximum Likelihood Blood Velocity Estimator Incorporating Properties of Flow Physics
}

Schlaikjer, Malene; Jensen, Jørgen Arendt

Published in:

I E E E Transactions on Ultrasonics, Ferroelectrics and Frequency Control

Link to article, DOI:

10.1109/TUFFC.2004.1268470

Publication date:

2004

Document Version

Publisher's PDF, also known as Version of record

Link back to DTU Orbit

Citation (APA):

Schlaikjer, M., \& Jensen, J. A. (2004). Maximum Likelihood Blood Velocity Estimator Incorporating Properties of Flow Physics. I E E E Transactions on Ultrasonics, Ferroelectrics and Frequency Control, 51(1), 80-92. https://doi.org/10.1109/TUFFC.2004.1268470

\section{General rights}

Copyright and moral rights for the publications made accessible in the public portal are retained by the authors and/or other copyright owners and it is a condition of accessing publications that users recognise and abide by the legal requirements associated with these rights.

- Users may download and print one copy of any publication from the public portal for the purpose of private study or research.

- You may not further distribute the material or use it for any profit-making activity or commercial gain

- You may freely distribute the URL identifying the publication in the public portal 


\title{
Maximum Likelihood Blood Velocity Estimator Incorporating Properties of Flow Physics
}

\author{
Malene Schlaikjer and Jørgen Arendt Jensen, Senior Member, IEEE
}

\begin{abstract}
The aspect of correlation among the blood velocities in time and space has not received much attention in previous blood velocity estimators. The theory of fluid mechanics predicts this property of the blood flow. Additionally, most estimators based on a cross-correlation analysis are limited on the maximum velocity detectable. This is due to the occurrence of multiple peaks in the crosscorrelation function. In this study a new estimator (CMLE), which is based on correlation (C) properties inherited from fluid flow and maximum likelihood estimation (MLE), is derived and evaluated on a set of simulated and in vivo data from the carotid artery. The estimator is meant for twodimensional (2-D) color flow imaging. The resulting mathematical relation for the estimator consists of two terms. The first term performs a cross-correlation analysis on the signal segment in the radio frequency (RF)-data under investigation. The flow physic properties are exploited in the second term, as the range of velocity values investigated in the cross-correlation analysis are compared to the velocity estimates in the temporal and spatial neighborhood of the signal segment under investigation. The new estimator has been compared to the cross-correlation (CC) estimator and the previously developed maximum likelihood estimator (MLE). The results show that the CMLE can handle a larger velocity search range and is capable of estimating even low velocity levels from tissue motion. The $\mathrm{CC}$ and the MLE produce incorrect velocity estimates due to the multiple peaks, when the velocity search range is increased above the maximum detectable velocity. The root-mean square error (RMS) on the velocity estimates for the simulated data is on the order of $7 \mathrm{~cm} / \mathrm{s}(14 \%)$ for the CMLE, and it is comparable to the RMS for the CC and the MLE. When the velocity search range is set to twice the limit of the $\mathrm{CC}$ and the MLE, the number of incorrect velocity estimates are 0 , 19.1, and $7.2 \%$ for the CMLE, CC, and MLE, respectively. The ability to handle a larger search range and estimating low velocity levels was confirmed on in vivo data.
\end{abstract}

\section{INTRODUCTION}

$\mathrm{M}$ EDICAL ultrasound imaging is used extensively for diagnosing diseases in the cardiovascular system (e.g., arterial stenosis, insufficient heart valve function). The distribution and magnitude of the blood velocities contain valuable information for the diagnosing. Estimates of the blood velocities can be obtained from a set of acquired RF-signals by proper processing of these. Over the years a

Manuscript received July 10, 2002; accepted August 8, 2003. This work was supported by grant 9700883, 9700563, and 5013-00-0025 from the Danish Science Foundation, grant 980018-311 from the Technical University of Denmark, and by B-K Medical A/S, Herlev, Denmark.

The authors are with Ørsted•DTU, Technical University of Denmark, DK-2800 Kgs. Lyngby, Denmark (e-mail: mas@oersted.dtu.dk). number of estimators have been proposed and used in various scanners. An exhaustive presentation of all these are out of the scope of this article, but they can be obtained from the literature [1], [2].

All estimators employ the fact that a shift in position of the scatterers causes a shift in time of interaction; thereby, causing a shift in position of the reflected echo in the acquired RF-signal [1]. For narrow-band signals, the time shift can be approximated by a phase shift between the sample values in two consecutively acquired RF-signals at a given depth, due to the oscillating nature of the signals. This feature is exploited in the autocorrelation estimator developed by Namekawa et al. [3] in 1982, and was further described by Kasai et al. [4] in 1985. The blood velocity is computed here from the phase shift [4]. The estimator is computationally very simple and has been very popular. An extension to use averaging over a number of samples in each line was proposed by Loupas et al. [5], [6]. The maximum detectable velocity is limited and is inversely proportional to the center frequency and the time between pulse emissions. Blood velocities above the limit are aliased. Other estimators have been developed to overcome this problem at the cost of computationally more demanding algorithms. This is now of less concern due to the advances within electronics. Several estimators have been developed, which determine the time shift itself. Among these are the cross-correlation estimator [7][9], the maximum likelihood estimator [10], [11], and the butterfly search technique [12], [13]. The aim of the processing is to find matching signal segments in consecutive RF-signals. A similarity investigation is performed by means of a cross-correlation or a variance analysis. The analysis is performed for a range of temporal shifts, and the location of the maximum or minimum value of the resulting function determines the time shift, and thereby the velocity for the segment under investigation. In principle, any search range can be investigated with these estimators, and thereby the limitations of the autocorrelation estimator can be overcome. With the cross-correlation based estimators, this is unfortunately not completely true. An upper bound on the search range usually has to be introduced to avoid detection of incorrect peaks in the crosscorrelation function [14]-[16], which limits the usability of estimators based merely on a cross-correlation analysis.

A general property of the currently proposed estimators is that the acquired RF-signals are split up in a number of segments, and the estimation then is performed on each of these segments separately. The aspect of temporal and spatial correlation between the velocities in the blood vessels 
(and thereby among the segments) has not received much attention. In a study by Forsberg et al. [17] temporal correlation is incorporated, as the estimator parameters are continuously varied according to the previously estimated velocity. In the current study the physical situation of fluid flow in a tubing system is exploited. The theory of nonturbulent flow physics states that the transitions between the velocity levels in time and space occur as a smooth and continuous function [18]. Given the estimated velocities in neighboring points (in time and space) of the segment under investigation, a priori knowledge on the velocity value in the segment is present. The basis for the velocity estimation can be improved, if this property is acknowledged and incorporated into the estimator. The limitations of the previously mentioned estimators, hopefully, then can be circumvented.

In this paper a new estimator, which incorporates the correlation property and is based on maximum likelihood theory, will be presented. The estimator is meant for 2D color flow imaging. The estimator uses the correlation property along with the cross-correlation analysis of the RF-signals. The aspect of temporal and spatial correlation is discussed in Section II. In Section III, the maximum likelihood estimator by Ferrara and Algazi [10], [11] and the new maximum likelihood estimator will be introduced. The main purpose of this paper is to present a new estimator; therefore, the majority of the paper contains the theory on the estimator. A selection of results is presented to verify that the estimator works and has its justification. The simulated and in vivo data from the carotid artery used for the performance evaluation are presented in Section IV, and the performance of the two estimators is evaluated in Section V. Section VI contains a discussion of the estimator, and the work is summed up in Section VII.

\section{iI. Correlation Between Velocities in Blood VESSEL}

The cardiovascular system acts as a piping system, in which the blood flows to and from the organs and tissues in the human body. Investigation of non-turbulent flow physics [18] reveals that the temporal and spatial correlation exist, so the velocities in a bounded spatial and temporal neighborhood are similar (not identical) in order to fulfill the correlation property. Therefore, a situation with a high-negative velocity surrounded in time and space by high-positive velocities is unlikely. In the human cardiovascular system, turbulent flow also is present e.g., at vessel bifurcations and the heart valves. More complex flow patterns and flow physics exist under these conditions. In the following, flow under non-turbulent conditions is considered to investigate the feasibility of incorporating flow physics. Turbulent flow is to be addressed in future work.

The correlation property is confirmable by inspection of the velocity profiles for the larger arteries (e.g., carotid and femoral arteries) [19]. Examples of the velocity profile across the radius at a number of time instances in the car-
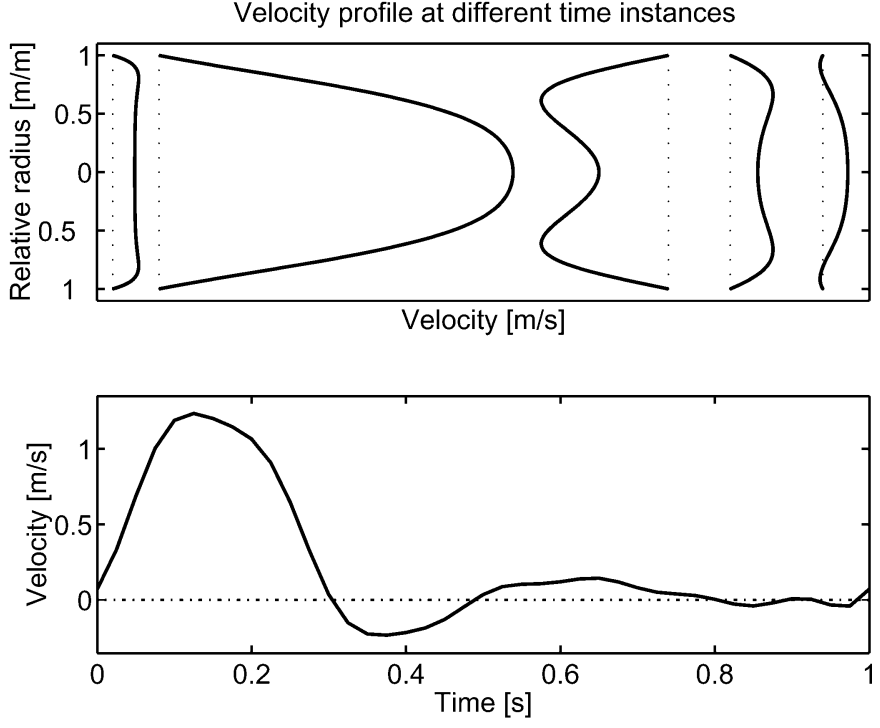

Fig. 1. Plots of velocity profiles in the femoral artery. Top: Velocity profiles along the diameter of the vessel for a number time instances in the cardiac cycle. The dotted, vertical lines indicate zero velocity. Bottom: Blood velocity as a function of time in the center of the femoral artery. Heart beat rate : 60 beats/minute.

diac cycle are plotted in the top plot in Fig. 1. The plots confirm the spatial correlation along the radial dimension. The temporal correlation is evident from the bottom plot in Fig. 1. Therefore, the velocity variations in a bounded region in time and space are limited. This introduces an a priori knowledge of the velocity in a given location, if the velocities in the neighborhood are known. It has to be similar in value to its temporal and spatial neighbors. This a priori knowledge should be exploited and incorporated in a blood velocity estimator. At this point it is important to stress that the variation patterns and the amplitude of velocity changes in time and space vary for the different vessels in the cardiovascular system. This also is evident in the plots. In most scanners today, the blood velocities are estimated at discrete time instances (controlled by frame rate and the recording of the individual RF-signals) on a discrete spatial grid. Under these circumstances, the velocities are not identical in a neighborhood as the velocities are not estimated at the same time instant. They are similar and, thereby, correlated when the individual estimates are not far apart in time and space. This is the case in medical ultrasound imaging with the current capabilities on frame rate and image resolution. Therefore, the level of temporal and spatial correlation among the neighboring values of the blood velocities are dependent on temporal and spatial resolution and must be accounted for in the estimator. So, despite the discrete nature of blood velocity estimation, the variations in time and space of the velocities will be limited and should be exploited in the estimator. The aim is to develop an estimator based on maximum likelihood theory, and a probability density function must be determined to express the correlation property. An estimate of the velocity $v$ in the segment under investigation is obtained through maximization of the probability density. 
A range of velocity values will be investigated. The likelihood of each velocity will be determined, and the most likely velocity will be the velocity estimate for the segment under investigation. It is the velocity projected onto the beam axis that is estimated. The correct velocity is obtained by correcting for the projection angle between the beam axis and the vessel. The probability density function, $p(v)$, will be split up in two terms: $p(v)=p_{a}(v) p_{\mathrm{RF}}(v)$. The a priori knowledge given by the correlation property is contained in $p_{a}$, whereas the information extractable from the acquired RF-signals in the segment under investigation is described by $p_{\mathrm{RF}}(v)$. The determination of $p_{a}(v)$ is the focus of this section, and the derivation of $p_{\mathrm{RF}}(v)$ is described in Section III.

A mathematical measure of similarity should be derived to describe the correlation property, and the probability density $p_{a}(v)$ determined herefore. A characteristic of two similar values is that the difference is small and limited. A difference comparison between the velocity in the point under investigation $v(l, s, f)$ and its neighbors $v(l+i, s+$ $j, f+k)$ determines similarity:

$$
D(i, j, k)=\frac{v(l+i, s+j, f+k)-v(l, s, f)}{\Delta(i, j, k)},
$$

where $l$ represents the line number in the image, $s$ is the segment number in the line, and $f$ is the frame number. A normalization variable $\Delta(i, j, k)$ has been introduced in the difference computation. It determines the distance in time or space between neighbors and, therefore, accounts for a given resolution in the velocity profiles. The variables $i$ and $j$ determine the bounded spatial neighbors, and they take values in the range $[-1 ; 1]$. The variable $k$ can take only the values -1 and 0 , as only velocity estimates from the present and previous frame are available at the time of the evaluation of segment $s$ in line $l$ in frame $f$. Only certain combinations of $i, j$, and $k$ will be used, and a discussion and definition of these follows later. At this point it is assumed that the differences between the velocities are small and limited. This assumption is investigated for the blood flow in the human vessels through a histogram analysis of the differences for a given velocity profile. The differences $D$ along the acoustical (axial) axis for $(i, j, k)=(0,1,0)$, the lateral axis $(i, j, k)=(1,0,0)$, and the temporal axis $(i, j, k)=(0,0,-1)$ have been determined for the femoral and carotid arteries throughout a full cardiac cycle. With these choices of $(i, j, k)(1)$ represents the spatial and temporal derivatives. The measure along the lateral and axial directions determines the shear rate between spatial neighbors. The acceleration is determined in the temporal direction. A number of 2-D velocity images equivalent to the color flow maps (CFM) on a scanner were produced. Thereby, the variations due to the differences in acquisition time of the individual RF-signals for each image line are accounted for. The velocity values were determined by defining a vessel with a physical realistic radius. For the femoral and carotid arteries Womersley's blood flow model [19] determines the velocity at any position as a function of time. A typical time sequence for acquisition of RF-

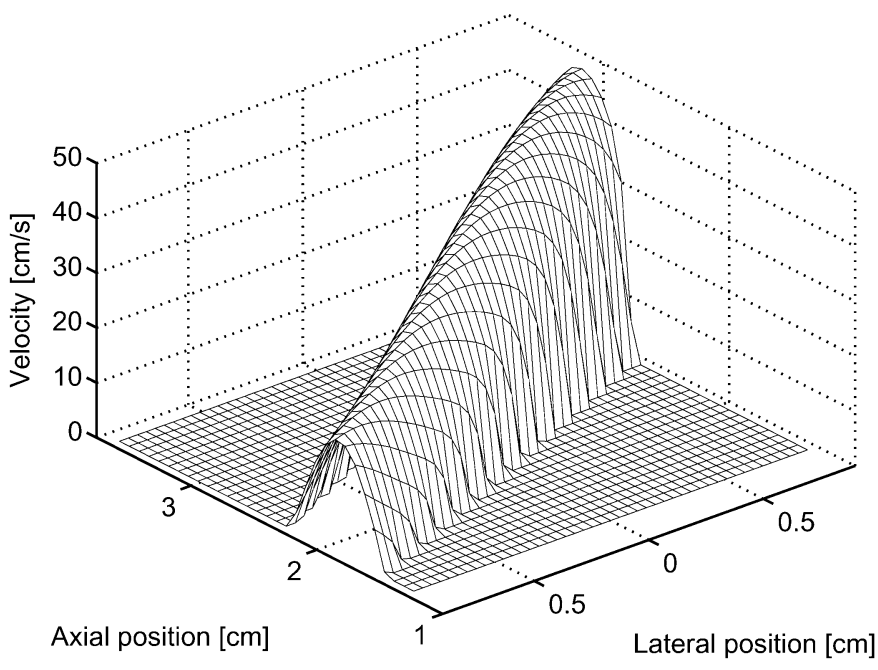

Fig. 2. Example of a 2-D velocity image for CFM imaging in the systolic phase.

signals for color flow imaging was used to determine the time values, which were inserted in the model to obtain the individual lines in a 2-D velocity image. A set of images equal to the time span of a cardiac cycle were generated, and an example of a $2-\mathrm{D}$ velocity image (imaged as a surface plot) is plotted in Fig. 2. The frame rate for the given scan setup is 10 frames/s, and the distance between lines and axial pixel centers are $0.4 \mathrm{~mm}$ and $0.8 \mathrm{~mm}$, respectively. Histogram plots of the differences for the carotid and femoral arteries are shown in Fig. 3. The plots verify that the temporal and spatial velocity variations between neighbors are limited. The course of the distributions can be explained through inspection of the flow profiles and the variations from frame to frame. The high, negative difference values, which occur in the temporal density of the carotid artery, resemble the velocity changes when the present and previous frame contain part of the systolic and the diastolic phase, respectively. The positive velocity differences occur during the diastolic phase. The latter has a longer temporal duration; therefore, small changes are seen more often in the cardiac cycle. The plots represent one realization of the flow profile across the vessel over a cardiac cycle for the current choice of image size and resolution. To allow for some variation and to lower the complexity of the estimator, the temporal and spatial density functions are approximated by a Gaussian distribution with zero mean. The statistical distribution for the measure of similarity, therefore, is set as follows:

$$
p_{a}(v(l, s, f))=\frac{1}{Z} \exp \left(-\frac{\zeta_{1} D_{1}(l, s, f)}{\sigma_{1}^{2}}-\frac{\zeta_{2} D_{2}(l, s, f)}{\sigma_{2}^{2}}\right)_{(2)}
$$

where $Z$ is a normalization constant, which assures that $p_{a}(v)$ sums up to one when integrated. The parameters $\zeta_{1}$ and $\zeta_{2}$ are scaling factors, and $\sigma_{1}^{2}$ and $\sigma_{2}^{2}$ are the variances of the temporal and spatial distributions. The subscript $a$ is used to indicate that the defined density determines an a priori knowledge of the situation under investigation (in this case the estimation of the velocity in a given spatial 

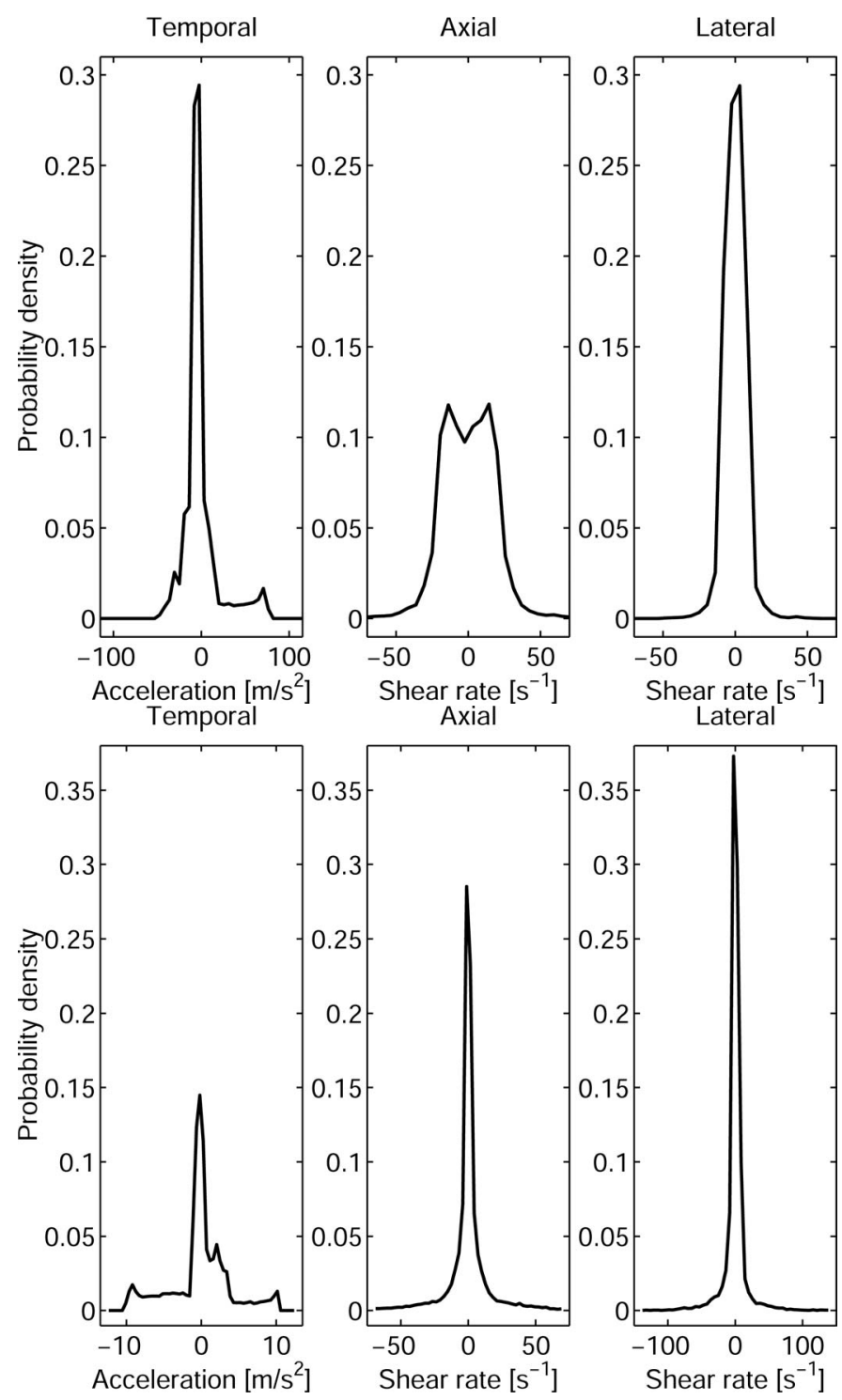

Fig. 3. The temporal, lateral, and axial probability densities of the differences for the carotid artery (top row) and femoral artery (bottom row) on simulated 2-D CFM images.

point). The terms $D_{1}$ and $D_{2}$ contain the similarity investigation along the temporal and spatial direction. The splitting of the similarity investigation in two terms makes it possible to incorporate differences in the levels of spatial and temporal correlation. Before defining $D_{1}$ and $D_{2}$, the neighborhood available, when estimating the velocity in $(l, s, f)$ must be determined. The sequence, which determines the acquisition order of the RF-data for velocity estimation across the image, varies among scanners, the scan setup, the range of velocities to be imaged, etc. The choice of acquisition sequence and processing scheme determine which spatial neighbors are available and can be used at the time of estimation of the velocity in $(l, s, f)$. In this study the following processing scheme is assumed in the medical ultrasound scanner:

- the set of RF-lines for each lateral position are processed after each other (line by line from left to right), and
- each line is split up in a number of segments, and the segments are processed after each other (from first to last segment).

With this choice, only two velocity estimatesrepresenting two spatial neighbors along the axial and lateral directions - within the same frame are available when the estimation is carried out for position $(l, s, f)$. The velocity estimates are: $\hat{v}(l-1, s, f)$ and $\hat{v}(l, s-1, f)$. Velocity estimates from the previous frame are used to make a complete neighborhood, which contains information around the segment under investigation. The neighborhood of velocity estimates $V$ becomes: $V=[\hat{v}(l-1, s, f), \hat{v}(l+1, s, f-$ $1), \hat{v}(l, s-1, f), \hat{v}(l, s+1, f-1), \hat{v}(l, s, f-1)]$. Note that the velocities in $V$ are themselves estimates, which have been obtained with the estimator in a previous step in the processing of the acquired RF data. A full neighborhood does not exist for the first and last segment in each line, the first and last line in the image, and the first frame. Therefore, the similarity investigation cannot be carried out and is omitted in the estimation for these situations. Based on the available estimates, $V$, the difference terms, $D_{1}$ and $D_{2}$, are defined as:

$$
\begin{aligned}
D_{1}(l, s, f)= & \left(\hat{v}(l, s, f-1)-v_{p}(l, s, f)\right)^{2}+(\hat{v}(l+1, s, f-1) \\
- & \left.v_{p}(l, s, f)\right)^{2}+\left(\hat{v}(l, s+1, f-1)-v_{p}(l, s, f)\right)^{2}, \\
D_{2}(l, s, f)= & \left(\hat{v}(l-1, s, f)-v_{p}(l, s, f)\right)^{2} \\
& +\left(\hat{v}(l, s-1, f)-v_{p}(l, s, f)\right)^{2},
\end{aligned}
$$

where $v_{p}$ represents the velocity currently being investigated as the velocity estimate for segment $s$ in line $l$ in frame $f$. All estimates from the previous frame irrespective of spatial position - are gathered in $D_{1}$. In $D_{2}$ the available spatial neighbors within the same frame are used. The normalization variable in (1) will be contained in the following in $\sigma_{1}$ and $\sigma_{2}$. A range $(p=\{1, N\})$ of (discrete) velocity values are investigated for each segment. At this point the a priori knowledge present due to the correlation property has been mathematically quantified and a density determined, which can express if the velocity $v_{p}$ is likely given the velocities in the neighborhood $V$. The acquired RF-data along each image line also contain valuable information for the estimation of the velocity, and the incorporation of this and the derivation of $p_{\mathrm{RF}}$ is the topic of the subsequent section.

\section{Maximum Likelihood Velocity Estimation}

The theory of maximum likelihood detection and estimation have been developed and used in radars [20], [21]. The techniques also are applicable for blood velocity estimation and will be exploited in this study. In Section III-A an estimator based on the maximum likelihood theory is derived [10], [11], and the incorporation of the correlation property follows in Section III-B. 


\section{A. Wideband Maximum Likelihood Estimator}

A characteristic signal, $\sqrt{E} s(t)$, emitted by an object (e.g., air-plane or blood scatterer) is a sufficient basis for the detection and estimation of location and velocity of the object. The parameter $E$ represents the signal energy, and it is assumed that $\int_{0}^{T_{s}} s^{2}(t) d t=1$. The variable $T_{s}$ represents the temporal length of the characteristic signal. Use of a sensor (e.g., transducer) makes it possible to acquire the signal and perform an analysis to determine the features of the object. As the presence of the object is not known a priori, the acquired signal, $r_{c}(t)$, either contains noise, $n$, only or the emitted signal with noise added. Two situations - equal to two hypotheses $\left(H_{0}, H_{1}\right)$ in statistics - can exist:

$$
r_{c}(t)= \begin{cases}\sqrt{E} s\left(t+\tau_{1}\right)+n(t) & 0 \leq t \leq T: H_{1} \\ n(t) & 0 \leq t \leq T: H_{0}\end{cases}
$$

where $T$ represents the temporal length of an observation. The subscript $c$ indicates that the signal is represented in complex form. The temporal location of the characteristic signal within the acquired signal is described by the temporal variable $\tau_{1}$, which value is unknown. Therefore, the acquired signal $r_{c}(t)$ must be split up in a number of signal segments, $r_{s}(t, \tau)$, of length $T_{s},\left(T_{s}<T\right)$. The aim of the subsequent analysis is to detect, if any of the segments contain the characteristic signal, so the value of $\tau_{1}$ can be determined. Thereby the presence and location of the object are estimated. The velocity can be estimated by acquiring a number of signals $r_{c}(t)$, and determine $\tau_{1}$ for each. The temporal shifts, $\tau_{1}$, between the different acquired lines along with the sound speed give the basis for an estimate of the velocity of the object. The information present in each signal segment, $r_{s}(t, \tau)$, can be compressed into a set of coefficients, $q_{j}$, based on a series expansion:

$$
\begin{gathered}
r_{s}(t, \tau)=\lim _{K \rightarrow \infty} \sum_{j=1}^{K} q_{j} \psi_{j}(t), \\
0 \leq t \leq T_{s},
\end{gathered}
$$

where $\psi_{j}$ are a set of orthonormal functions. If $\psi_{1}$ is equal to $s(t)$, the coefficient $q_{1}$ become:

$$
q_{1}= \begin{cases}\int_{0}^{T_{s}}(\sqrt{E} s(t)+n(t)) s^{*}(t) d t & =\sqrt{E}+n_{1}: H_{1} \\ \int_{0}^{T_{s}} n(t) s^{*}(t) d t & =n_{1}: H_{0}\end{cases}
$$

All other $q_{j}(j>1)$ will contain no information related to $s(t)$, as all $\psi_{j}$ are orthogonal to $s(t)$. The information needed to perform the detection and estimation is fully contained in $q_{1}$, and a set of $q_{1}$ values are determined for each value of $\tau$. The computation of $q_{1}$ equals a correlation analysis between $r_{s}(t, \tau)$ and $s(t)$, so $q_{1}(\tau)$ tells how alike these signals are. The theory on maximum likelihood estimation [20], [21] predicts that the estimate of $\tau_{1}$ is de- termined by computing $q_{1}$ for a range of $\tau$ values. The estimate is found, where the likelihood ratio:

$$
\operatorname{LR}\left(Q_{1}(\tau)\right)=\frac{p_{q_{1} \mid H_{1}}\left(Q_{1} \mid H_{1}\right)}{p_{q_{1} \mid H_{0}}\left(Q_{1} \mid H_{0}\right)},
$$

takes its maximum value:

$$
\hat{\tau}_{1}=\underset{\tau}{\arg \max }\left(L R\left(Q_{1}(\tau)\right)\right) .
$$

The variable $Q_{1}$ represents an observation of $q_{1}$ for a given value of $\tau$, and $p_{q_{1} \mid H_{0}}$ and $p_{q_{1} \mid H_{1}}$ are the probability density functions under $H_{0}$ and $H_{1}$, respectively. The likelihood ratio represents a comparison of the probability values, and the ratio value expresses which hypothesis is more likely.

The above method is applicable for blood velocity estimation, although the characteristic signal is not known a priori. In medical ultrasound, a signal is emitted by the transducer and propagates in tissue. The signal is scattered by the individual scatterers distributed in space. The acquired RF-signals, therefore, consist of the sum of reflected contributions from the scatterers. As the composition of the tissue structures (e.g., blood, vessels, fat, liver, or kidney tissue) is not known a priori, it is not possible to predict the course of the acquired RF-signal. Still, a limited group (in space) of scatterers generate a characteristic pattern in the RF-signal, which will show in successive acquired RF-signals along the same line [10], [22]. If the scatterers are moving between acquisitions, the temporal position of the pattern will change according to the velocity at a constant rate, as long as the successive RF-signals are acquired within a time window of few milliseconds. The latter requirement is fulfilled in commercially available ultrasound scanners. The need for a characteristic signal to perform the correlation analysis in (6) can be obtained by using the characteristic patterns in the RF-signal acquired first. The first RF-signal, therefore, is split up in a number of segments, and the signal in each of these is used as the characteristic signal to determine the position of the pattern in the successive RF-signals. The processing and the resulting estimate of the temporal shift is performed for each of the segments in the RF-signals. Thereby, the computation of $q_{1}$ becomes a correlation analysis between a segment in the first line and a temporally shifted segment in a successive line, $l$ :

$$
\begin{aligned}
& q_{1, l}\left(v_{p}\right)= \\
& \sum_{k=0}^{N_{s}-1} r_{s}\left(1, k+i_{\text {seg }} N_{s}\right) r_{s}\left(l, k+i_{\text {seg }} N_{s}+m \cdot(l-1)\right),
\end{aligned}
$$

where $m$ gives the temporal shift in position of the segments in the RF-signals in number of samples and is a function of the investigated velocity $v_{p}$. The parameter $i_{\text {seg }}$ determines the segment number, and $N_{s}$ tells the number of samples within this segment. The number of lines available for the analysis are referred to by the parameter $N_{l}$, and thereby, $N_{l}$ values of $q_{1}$ are obtained from each correlation analysis of one segment for one choice of the temporal 
shift. In the following each of these observations are referred to as $Q_{1, l}$. The probability of $q_{1, s}=q_{1,1}+\cdots+q_{1, N_{l}}$ is [20], [21], [23], [24]:

$$
\begin{aligned}
& p_{q_{1, s} \mid H_{1}}\left(Q_{1, s} \mid H_{1}\right)= \\
& \frac{1}{\pi\left(E+N_{0}\right) N_{l}} \exp \left(-\frac{\left|Q_{1, s}\left(v_{p}\right)\right|^{2}}{\left(E+N_{0}\right) N_{l}}\right),
\end{aligned}
$$

where $N_{0}$ is the energy of the noise. The probability density of $q_{1, s}$ under $H_{0}$ is:

$$
p_{q_{1, s} \mid H_{0}}\left(Q_{1, s} \mid H_{0}\right)=\frac{1}{\pi N_{0} N_{l}} \exp \left(-\frac{\left|Q_{1, s}\left(v_{p}\right)\right|^{2}}{N_{0} N_{l}}\right) .
$$

As the probability density of $q_{1, s}$ is known under $H_{0}$ and $H_{1}$, the likelihood ratio can be derived:

$$
\begin{aligned}
\operatorname{LR}\left(Q_{1, s}\left(v_{p}\right)\right) & =\frac{p_{q_{1, s} \mid H_{1}}\left(Q_{1, s} \mid H_{1}\right)}{p_{q_{1, s} \mid H_{0}}\left(Q_{1, s} \mid H_{0}\right)} \\
& =\frac{\left(\pi\left(E+N_{0}\right) N_{l}\right)^{-1} \exp \left(-\frac{\left|Q_{1, s}\left(v_{p}\right)\right|^{2}}{\left(E+N_{0}\right) N_{l}}\right)}{\left(\pi N_{0} N_{l}\right)^{-1} \exp \left(-\frac{\left|Q_{1, s}\left(v_{p}\right)\right|^{2}}{N_{0} N_{l}}\right)}
\end{aligned}
$$

By taking the logarithm and rearranging the terms, the following relation is obtained:

$$
\begin{aligned}
\ln \left(L R\left(Q_{1, s}\left(v_{p}\right)\right)\right)= & \ln \left(\frac{N_{0}}{E+N_{0}}\right) \\
& +\frac{E}{\left(E+N_{0}\right) N_{0} N_{l}}\left|Q_{1, s}\left(v_{p}\right)\right|^{2}
\end{aligned}
$$

The parameters $N_{0}, E$, and $N_{l}$ are constants. They only contribute with an offset value and a scaling of $\left|Q_{1, s}\left(v_{p}\right)\right|^{2}$, so $\left|Q_{1, s}\left(v_{p}\right)\right|^{2}$ represents the sufficient statistic (the measure) that is needed to perform an estimation of $v_{p}$. If $\left|Q_{1, s}\left(v_{p}\right)\right|^{2}$ is computed [using (9) and summing the $N_{l}$ values] for the range of velocities, the maximum of $\left|Q_{1, s}\left(v_{p}\right)\right|^{2}$ gives the estimate of the (average) velocity for a group of scatterers in the segment under investigation:

$$
\hat{v}(l, s, f)=\underset{v_{p}}{\arg \max }\left(\left|Q_{1, s}\left(v_{p}\right)\right|^{2}\right) .
$$

This estimator represents the approach of the wideband maximum likelihood estimator by Ferrara and Algazi (1991) [10], [11]. The term wideband refers to the emission of a wideband excitation pulse, which will improve the basis for the correlation analysis. In the following this estimator will be referred to as the MLE.

\section{B. Extended Maximum Likelihood Estimator}

In the above estimator, the a priori knowledge of the spatial and temporal correlation on the velocity is not incorporated. The a priori knowledge can be included by expanding the density under $H_{1}$ with $p_{a}$ and thereby generating the joint probability density:

$$
p\left(q_{1}, v_{p}(l, s, f)\right)=p_{a}\left(v_{p}(l, s, f)\right) p_{q_{1} \mid H_{1}} .
$$

By inserting (2) and (10) into this equation the probability density under $H_{1}$ becomes:

$$
\begin{aligned}
& p_{q_{1, s} \mid H_{1}}\left(Q_{1, s}, v_{p} \mid H_{1}\right)= \\
& \frac{1}{Z} \frac{1}{\pi\left(E+N_{0}\right) N_{l}} \exp \left(-\frac{\zeta_{1} D_{1}(l, s, f)}{\sigma_{1}^{2}}-\frac{\zeta_{2} D_{2}(l, s, f)}{\sigma_{2}^{2}}\right) . \\
& \quad \exp \left(-\frac{\left|Q_{1, s}\left(v_{p}\right)\right|^{2}}{\left(E+N_{0}\right) N_{l}}\right) .
\end{aligned}
$$

The probability density of $q_{1, s}$ under $H_{0}$ is unchanged:

$$
p_{q_{1, s} \mid H_{0}}\left(Q_{1, s}, v_{p} \mid H_{0}\right)=\frac{1}{\pi N_{0} N_{l}} \exp \left(-\frac{\left|Q_{1, s}\left(v_{p}\right)\right|^{2}}{N_{0} N_{l}}\right)
$$

According to the discussion in Section III-A, an estimator can be obtained by determining the likelihood ratio. When both the a priori and the signal specific information are included, the likelihood ratio takes the form shown in (18) (see next page). If all the exponentials are combined into one, and the logarithm of LR is computed, the following relation is obtained:

$$
\begin{aligned}
\ln \left(L R\left(Q_{1, s}, v_{p}\right)\right)= & \ln \left(\frac{N_{0}}{Z\left(E+N_{0}\right)}\right) \\
& -\frac{\zeta_{1} D_{1}(l, s, f)}{\sigma_{1}^{2}}-\frac{\zeta_{2} D_{2}(l, s, f)}{\sigma_{2}^{2}} \\
& -\left|Q_{1, s}\left(v_{p}\right)\right|^{2} \frac{1}{N_{l}}\left(\frac{1}{E+N_{0}}-\frac{1}{N_{0}}\right) \\
= & \ln \left(\frac{N_{0}}{Z\left(E+N_{0}\right)}\right)-\frac{\zeta_{1} D_{1}(l, s, f)}{\sigma_{1}^{2}} \\
& -\frac{\zeta_{2} D_{2}(l, s, f)}{\sigma_{2}^{2}} \\
& +\frac{1}{N_{l}} \frac{E}{\left(E+N_{0}\right) N_{0}}\left|Q_{1, s}\left(v_{p}\right)\right|^{2}
\end{aligned}
$$

The variables $\zeta_{1}$ and $\zeta_{2}$ are related to the energy content in the RF-signals and will be defined as:

$$
\begin{aligned}
& \zeta_{1}=\kappa_{1} \frac{E\left(E+N_{0}\right)}{N_{0}}, \\
& \zeta_{2}=\kappa_{2} \frac{E\left(E+N_{0}\right)}{N_{0}},
\end{aligned}
$$

where $\kappa_{1}$ and $\kappa_{2}$ are a new set of scale factors. These definitions are introduced in (19). By rearranging terms, ignoring the first term and common constants for the last terms, the sufficient statistic in the form of the likelihood ratio $\left(L R_{S}\right)$ can be expressed as:

$$
\begin{aligned}
\ln \left(L R_{S}\left(Q_{1, s}, v_{p}\right)\right) & =\frac{1}{N_{l}\left(E+N_{0}\right)^{2}}\left|Q_{1, s}\left(v_{p}\right)\right|^{2} \\
& -\frac{\kappa_{1} D_{1}(l, s, f)}{\sigma_{1}^{2}}-\frac{\kappa_{2} D_{2}(l, s, f)}{\sigma_{2}^{2}} .
\end{aligned}
$$

The values of the variables $\kappa_{1}, \kappa_{2}, \sigma_{1}^{2}$, and $\sigma_{2}^{2}$ should be determined and are dependent on the spatial and temporal resolution. The exact values for the individual variables 


$$
\begin{aligned}
L R\left(Q_{1, s}, v_{p}\right) & =\frac{p_{q_{1, s} \mid H_{1}}\left(Q_{1, s} \mid H_{1}\right)}{p_{q_{1, s} \mid H_{0}}\left(Q_{1, s} \mid H_{0}\right)} \\
& =\frac{\left(Z \pi\left(E+N_{0}\right) N_{l}\right)^{-1} \exp \left(-\frac{\zeta_{1} D_{1}(l, s, f)}{\sigma_{1}^{2}}-\frac{\zeta_{2} D_{2}(l, s, f)}{\sigma_{2}^{2}}\right) \exp \left(-\frac{\left|Q_{1, s}\left(v_{p}\right)\right|^{2}}{\left(E+N_{0}\right) N_{l}}\right)}{\left(\pi N_{0} N_{l}\right)^{-1} \exp \left(-\frac{\left|Q_{1, s}\left(v_{p}\right)\right|^{2}}{N_{0} N_{l}}\right)} .
\end{aligned}
$$

\section{Step A}

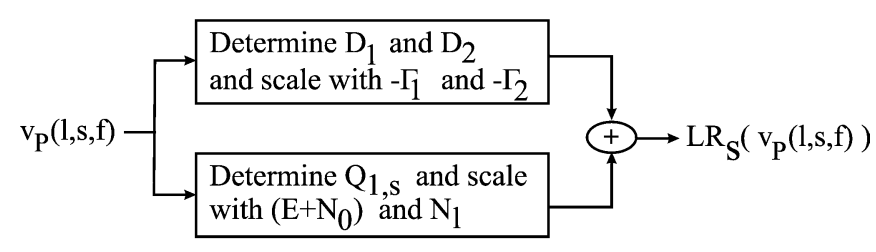

\section{Step B}

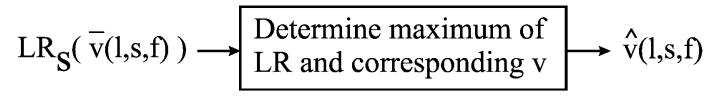

Fig. 4. Flow chart showing the steps to be performed for each segment to obtain a velocity estimate.

will not be considered in this context. Instead the variables are combined as:

$$
\begin{aligned}
& \Gamma_{1}=\kappa_{1} / \sigma_{1}^{2}, \\
& \Gamma_{2}=\kappa_{2} / \sigma_{2}^{2} .
\end{aligned}
$$

The mathematical relation describing the estimator based on maximum likelihood theory, which includes the spatial and temporal correlation among the velocities in a bounded region, is:

$$
\begin{aligned}
\hat{v}(l, s, f)= & \underset{v_{p}}{\arg \max }\left(\ln \left(L R_{S}\left(Q_{1, s}, v_{p}\right)\right)\right) \\
= & \underset{v_{p}}{\arg \max }\left(\frac{1}{N_{l}\left(E+N_{0}\right)^{2}}\left|Q_{1, s}\left(v_{p}\right)\right|^{2}\right. \\
& \left.-\Gamma_{1} D_{1}(l, s, f)-\Gamma_{2} D_{2}(l, s, f)\right) .
\end{aligned}
$$

The equation consists of three terms. The first term describes the correlation analysis between signal segments and a normalization with signal energy. The last two terms handle the similarity investigation in time and space. The processing involved in the estimation proceeds in two steps as shown in Fig. 4. The variable $\bar{v}$ represents the set of $p$ discrete velocity values being investigated. Step A, therefore, is repeated $p$ times before the velocity estimate $\hat{v}(l, s, f)$ can be determined in Step B. The processing has to be performed for each segment in the acquired RF-signal to determine the velocity distribution throughout the region scanned and for a range of velocities. The estimator, thereby, becomes computationally demanding compared to the simple autocorrelation estimator, but the limitations on the maximum velocity detectable should be eliminated and the physics of the in vivo situation has been accounted for in the estimation. The latter should improve the estimation, as it becomes possible to express a measure of belief in an estimate as part of the estimation. For most other estimators, the variance of the estimate can be determined, but it is not possible to use it and thereby benefit from this knowledge in the estimation.

An estimator, which is based on maximum likelihood theory and incorporates the temporal and spatial correlation between the blood velocities, has been derived in the above. It will be named the CMLE, where the $\mathrm{C}$ refers to incorporation of the correlation property. The performance of the estimator is influenced by decorrelation of the patterns in successive RF-signals and the accuracy on the neighboring velocity estimates. The latter influences the terms $D_{1}$ and $D_{2}$. The decorrelation arises, as the individual scatterers move at different velocities, so the distance traveled between two acquisitions will be different. The summed reflected responses, therefore, will not give identical patterns in two consecutive lines. Within the time frame of a few milliseconds this effect is less dominant, and the estimator described can perform blood velocity estimation. The estimator is expected to perform best on wideband excitation pulses, as these give the best basis for the cross-correlation analysis. The performance evaluation on the simulated data uses this, whereas the performance on in vivo data is performed on narrow band pulses. The latter is due to the settings of the B-K 2102 Medical A/S (Herlev, Denmark) scanner used.

\section{DATA}

The ability of the two maximum likelihood estimators to perform blood velocity estimation has been investigated on simulated and in vivo data. An exhaustive evaluation of performance for all possible scenarios with respect to blood flow profile, signal-to-noise (SNR) ratios, image size and resolution, etc. is out of the scope for this paper. The main purpose is to show that the correlation property is of importance in blood velocity estimation and can be implemented in an estimator.

\section{A. Simulated Data}

Simulated data resembling RF-data acquired from the carotid artery have been generated with Field II [25], 
TABLE I

Choice of Parameters for the Simulated Data Used in the Performance Evaluation of the Blood Velocity Estimators.

\begin{tabular}{llr}
\hline Parameter & Symbol & Value \\
\hline Center frequency of pulse & $f_{0}$ & $5.0 \mathrm{MHz}$ \\
No. of elements & $N$ & 128 \\
No. of active elements & $N_{a}$ & 32 \\
Pulse length (in cycles) & $P_{l}$ & 1.5 \\
Geometric focus & $F_{g}$ & $17 \mathrm{~mm}$ \\
Pulse repetition frequency & $f_{\text {prf }}$ & $4 \mathrm{kHz}$ \\
Sampling frequency & $f_{s}$ & $40 \mathrm{MHz}$ \\
Sound velocity & $c$ & $1540 \mathrm{~m} / \mathrm{s}$ \\
Radius of vessel & $R_{v}$ & $3 \mathrm{~mm}$ \\
Angle between vessel & & \\
and acoustical axis & $\theta$ & $55^{\circ}$ \\
\hline
\end{tabular}

[26]. Any transducer array, any focusing and apodization scheme, and any excitation pulse can be handled with the program. The program simulates the linear propagation, and the Born approximation excluding multiple scattering is applied. A focusing and apodization scheme matching the setup of a B-K Medical A/S (Herlev, Denmark) 3535 scanner, which was connected to a linear array, has been implemented. A wideband excitation pulse (a Hanning weighted sinusoidal pulse of 1.5 cycles) was used. Womersley's blood flow model [19] was used to model the motion of the blood scatterers. Tissue motion due to the pulsation of the vessel walls has been incorporated to obtain data, which resembles the in vivo situation [22], [27]. The parameters for the current simulation are listed in Table I. One cardiac cycle (one second) has been simulated. The pulsing strategy alternates between acquiring one RFline for the B-mode image, then 8 RF-lines for the blood velocity estimation. The lateral extent of the CFM image equals half the imaging range of the transducer, so a frame rate of 10 frames/s is obtained for the CFM-mode image. The low frame rate is of no concern in the current study, as the aim is to evaluate the estimation abilities of the estimators rather than displaying many images. Gaussian noise is present in the data. Two data sets were generated with SNR ratios of 10 and $20 \mathrm{~dB}$, respectively.

\section{B. In Vivo Data}

The in vivo RF-data were acquired with a dedicated ultrasound sampling system [28] interfaced to a B-K Medical A/S (Herlev, Denmark) 2102 scanner. The focusing and apodization scheme along with the excitation pulse was controlled by the 2102 scanner. A linear array transducer was used, and a narrow band excitation pulse with a center frequency of $5 \mathrm{MHz}$ was emitted. The beamformed RF-signals were acquired by the sampling system, which works at a sampling frequency of $40 \mathrm{MHz}$ and a 12 bits resolution. Ten seconds of CFM data were acquired, corresponding to several cardiac cycles. Nine RF-signals are acquired along each line in the CFM image. The focus was at $1.8 \mathrm{~cm}$, and the frame rate was equal to 16 frames $/ \mathrm{s}$.
TABLE II

Performance Measures as a Function of $\Gamma_{1}$ and $\Gamma_{2}$.

\begin{tabular}{|c|c|c|c|c|c|c|c|}
\hline \multirow{2}{*}{\multicolumn{2}{|c|}{ RMS }} & \multicolumn{6}{|c|}{$\Gamma_{1}$} \\
\hline & & 0 & 1 & 2 & 3 & 4 & 5 \\
\hline \multirow{6}{*}{$\Gamma_{2}$} & 0 & 6.86 & 7.03 & 7.04 & 7.05 & 7.04 & 6.97 \\
\hline & 1 & 7.05 & 7.05 & 7.04 & 7.07 & 7.04 & 7.01 \\
\hline & 2 & 7.06 & 7.06 & 7.07 & 7.05 & 7.03 & 7.01 \\
\hline & 3 & 7.07 & 7.08 & 7.07 & 7.08 & 7.03 & 7.02 \\
\hline & 4 & 7.1 & 7.08 & 7.08 & 7.07 & 7.02 & 7.01 \\
\hline & 5 & 7.12 & 7.08 & 7.07 & 7.08 & 7.03 & 7.02 \\
\hline \multirow{2}{*}{\multicolumn{2}{|c|}{ Outliers }} & \multicolumn{6}{|c|}{$\Gamma_{1}$} \\
\hline & & 0 & 1 & 2 & 3 & 4 & 5 \\
\hline \multirow{6}{*}{$\Gamma_{2}$} & 0 & 3.61 & 0.4 & 0.12 & 0.09 & 0.09 & 0.12 \\
\hline & 1 & 0.64 & 0.12 & 0.06 & 0 & 0.06 & 0.09 \\
\hline & 2 & 0.15 & 0.06 & 0 & 0 & 0.03 & 0.06 \\
\hline & 3 & 0.09 & 0 & 0 & 0 & 0.03 & 0.06 \\
\hline & 4 & 0.06 & 0 & 0 & 0 & 0 & 0.06 \\
\hline & 5 & 0.03 & 0 & 0 & 0 & 0 & 0 \\
\hline
\end{tabular}

Velocity search range, $50 \mathrm{~cm} / \mathrm{s}$. Top, RMS error $([\mathrm{cm} / \mathrm{s}])$. The error on the first frame was excluded, as the estimates were obtained with the MLE. Bottom, number of estimates (\%) outside the velocity range from $-10 \mathrm{~cm} / \mathrm{s}$ to $60 \mathrm{~cm} / \mathrm{s}$.

\section{RESUlts}

Prior to the blood velocity estimation, the RF-signals (both simulated and in vivo data sets) were matched filtered [1] to minimize the noise content, and stationary echo canceling was performed with a second order high-pass filter [1]. The resolution on the discrete velocity search was set to $1 \mathrm{~cm} / \mathrm{s}$. The basis for determining a large range of velocities (e.g., low velocity at vessel wall and in tissue, and a high velocity in center of vessel) is then given.

\section{A. Determination of Estimator Parameters}

The scale parameters $\Gamma_{1}$ and $\Gamma_{2}$ have to be assigned a value prior to using the CMLE for estimation. A performance evaluation for a range of scale values was carried out on the simulated data. This is possible, as the true 2-D velocity image is known for every frame and, thereby, can be compared to the estimated $2-\mathrm{D}$ velocity profile. Two performance parameters have been defined for the evaluation. The true velocities span the range from $0 \mathrm{~cm} / \mathrm{s}$ to $50 \mathrm{~cm} / \mathrm{s}$, and estimates outside the range $-10 \mathrm{~cm} / \mathrm{s}$ and $60 \mathrm{~cm} / \mathrm{s}$ are considered outliers. The total number of these summed over the full velocity image and the number of frames available are used as the first performance measure. The RMS error on the velocity estimates inside the true velocity range is used as the second measure. The mean operation is performed over all frames. The performance measures are listed in Table II. The RMS error has not been normalized with the peak velocity of $0.49 \mathrm{~m} / \mathrm{s}$ in the systole. The RMS error does not vary much over the range of $\Gamma_{1}$ and $\Gamma_{2}$. The influence of the choice of the scale parameters show on the number of outliers. For a narrow range of values, no outliers are produced with the CMLE, and in the following $\Gamma_{1}$ and $\Gamma_{2}$ will both be set equal to 2 . 

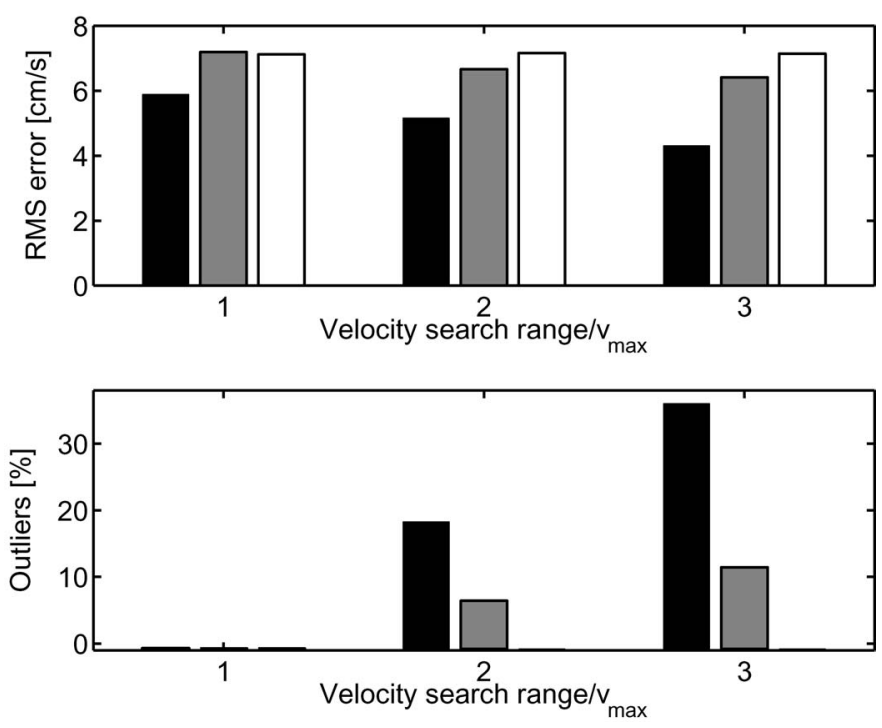

Fig. 5. Performance of the cross-correlation estimator (black), the MLE (gray), and the CMLE (white) on the simulated data resembling the carotid artery as a function of three velocity search ranges in the correlation computation. SNR is $20 \mathrm{~dB}$.

\section{B. Performance on Simulated Data}

As mentioned in the introduction, estimators based on cross-correlation usually are limited in the velocity search range. Multiple peaks in the cross-correlation function will occur at temporal shifts in the RF-signal, which equal one period of the oscillation: $1 / f_{0}$. Therefore, the upper bound on the velocity search range is usually set equal to half a period, which equals the maximum detectable velocity $v_{\max }$ with the autocorrelation estimator: $v_{\max }=c f_{\text {prf }} / 4 f_{0}=31 \mathrm{~cm} / \mathrm{s}$. The new estimator contains a cross-correlation analysis but additionally a term, which is related to the fluid properties. The question is, if the incorporation of the fluid properties can overcome the limitation on the velocity search range. The performance of the crosscorrelation estimator, the MLE, and the CMLE have been investigated for three different values of the search range, which are integer multiply of $v_{\max }$. The RMS error and the number of outliers were determined for each estimator for the three search ranges, and Fig. 5 shows the results for a SNR of $20 \mathrm{~dB}$. The peak velocity in the systole is $0.49 \mathrm{~m} / \mathrm{s}$, which results in relative RMS errors in the range from 8 to $15 \%$. The estimates in the first image frame, and the first and last line in the subsequent image frames, have been excluded in the computation of the performance measures, as a full neighborhood does not exist and the CMLE cannot carry out the estimation here. In these locations the MLE is used instead. The lowest RMS error is obtained with the cross-correlation estimator for any value of the search range. Unfortunately, the number of outliers increases significantly as soon as the search range is above $v_{\max }$. The MLE has the same problem with respect to the increasing number of outliers for increasing search range. The CMLE overcomes this problem completely. No estimates are outside the range $-10 \mathrm{~cm} / \mathrm{s}$ to $60 \mathrm{~cm} / \mathrm{s}$; therefore, the

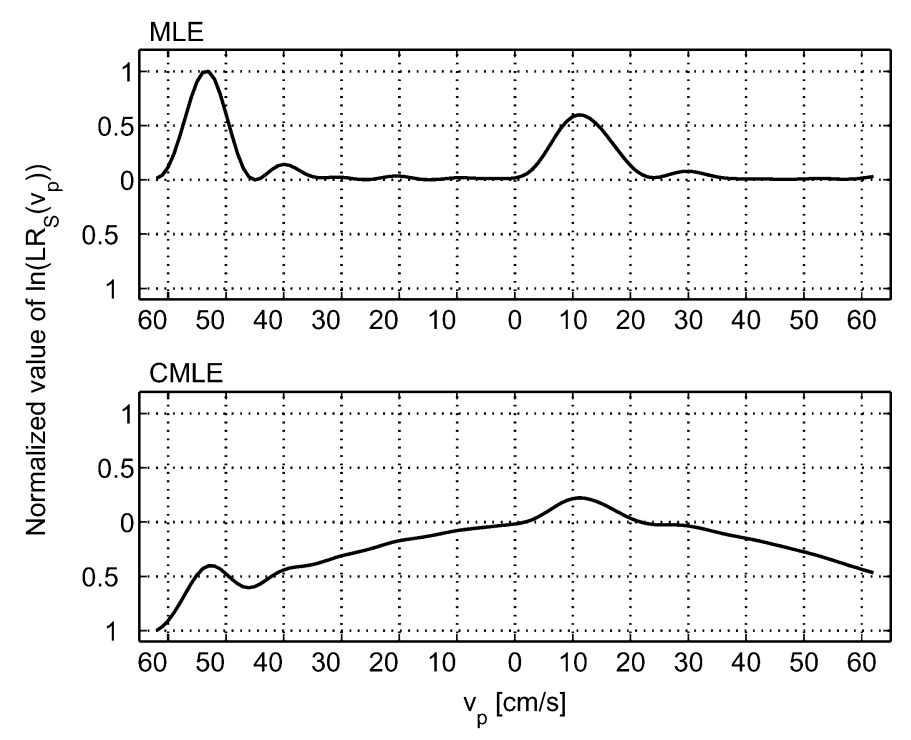

Fig. 6. The likelihood ratios for the MLE (top) and CMLE (bottom) for a range of velocities. The true velocity is $12.6 \mathrm{~cm} / \mathrm{s}$ for the spatial position $(0.5,3) \mathrm{cm}$, and the scaling parameters $\Gamma_{1}$ and $\Gamma_{2}$ are equal to $(2,2)$. Velocity search range: $2 * v_{\max }$.

problem of incorrect estimates due to the multiple peaks in the cross-correlation function does not occur. This is verified through inspection of the value of the likelihood ratio in (24) as a function of $v_{p}$ as shown in Fig. 6 for a given spatial position - in this case the position $0.5 \mathrm{~cm}$ laterally and $3 \mathrm{~cm}$ axially in the eighth image frame of 10 . With the MLE two peaks separated by $2 v_{\max }$ occur at $-50 \mathrm{~cm} / \mathrm{s}$ and $11 \mathrm{~cm} / \mathrm{s}$. The maximum of the likelihood ratio is at $-50 \mathrm{~cm} / \mathrm{s}$. An incorrect estimate of the velocity is produced, as the true velocity is $12.6 \mathrm{~cm} / \mathrm{s}$. The CMLE has only one peak at $11 \mathrm{~cm} / \mathrm{s}$, and therefore produces the most correct estimate of the two estimators. The reason is that the velocity $-50 \mathrm{~cm} / \mathrm{s}$ is far from the values of the neighboring velocities; therefore, the a priori density assigns a low probability to this velocity. This causes the value of the $L R_{S}$ to decrease, as the terms $\Gamma_{1} D_{1}$ and $\Gamma_{2} D_{2}$ take high values and therefore contribute to the sum in (24). The shape of the peak with the CMLE is less sharp than the peak obtained with the MLE, but it still stands out from the rest of the likelihood values, and it gives the basis for determining the estimate. The RMS error varies less than $1 \%$ for the three different search ranges. The same trends on the number of outliers show when the SNR in the simulation is lowered to $10 \mathrm{~dB}$. In this situation, the lowest RMS error is obtained with the CMLE independent of the choice of velocity search range, and the level is on the same order as seen in the $20 \mathrm{~dB}$ situation. The RMS error increases for both the cross-correlation estimator and the MLE. Therefore, the CMLE is capable of overcoming the limitations on the maximum detectable velocity as experienced with the autocorrelation, the cross-correlation, and the maximum likelihood estimators.

A typical example of the estimated velocity profiles is presented in Fig. 7 along with the true velocity profile. The plot corresponds to a color flow map on a scanner, in 


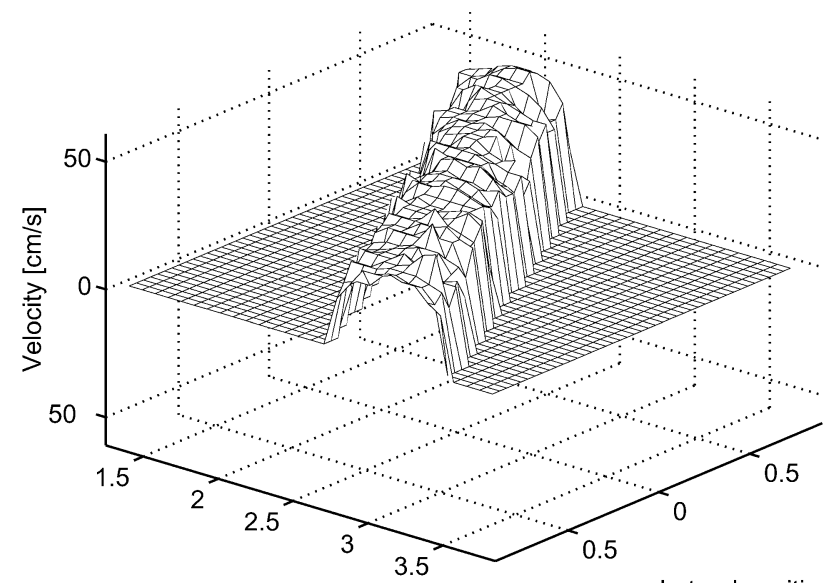

Lateral position $[\mathrm{cm}]$

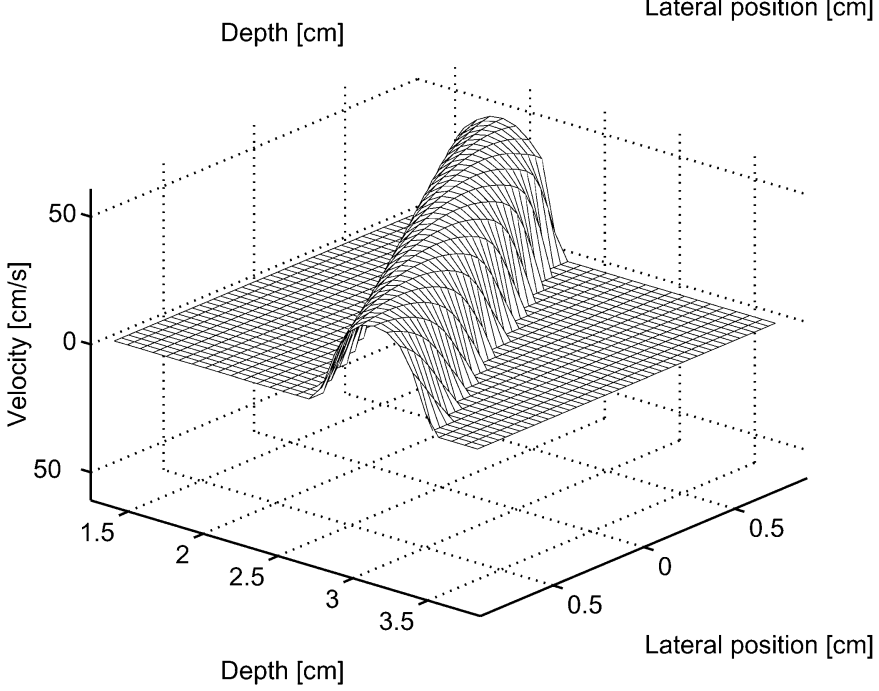

Fig. 7. Estimated (top) and true (bottom) velocity profile. Estimation performed with the CMLE on the simulated data, in which the SNR is $20 \mathrm{~dB}$. Data are from the systole of the cardiac cycle. Velocity search range: $2 * v_{\max }$.

which the gray-tone B-mode image has been left out. The usual color-coding has been replaced by actual velocity values. In Fig. 8 one line in the image has been plotted as a function of depth. The two plots in the top show the true velocity (solid), and the estimates with the MLE (dashed) and the CMLE (dotted), when the velocity search range equals $1 * v_{\max }$ (a) and $2 * v_{\max }$ (b), respectively. The vessel lies in the depth range between $1.48 \mathrm{~cm}$ to $2.35 \mathrm{~cm}$. The limitation on search range for the MLE is clearly visible. A comparison between the true and the estimated profiles reveal that the velocities at the vessel border are overestimated, and a more flat profile is obtained in the center of the vessel. But the velocity range of the estimates comes close to the true values. This result represents the performance generally seen for all frames throughout the cardiac cycle. Inspection of the computed estimates outside the vessel reveals a second advantage of the CMLE. More estimates are computed, which take values close to $0 \mathrm{~cm} / \mathrm{s}$. This effect is most pronounced in the systolic phase of the cardiac cycle, in which some tissue motion occurs due to the pulsation of the vessel walls. The level of motion is low (a)

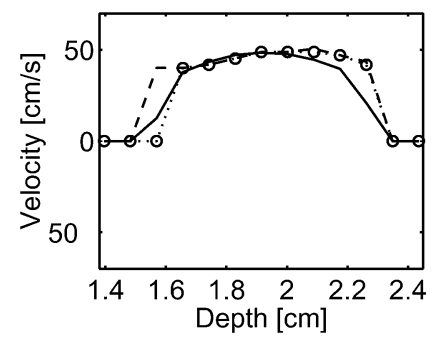

(c)

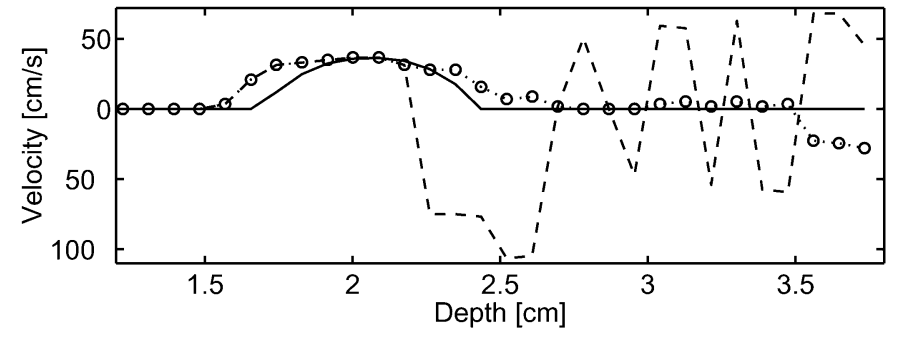

Fig. 8. The velocity estimates with the MLE (dashed), CMLE (dotted), and the true velocities (solid) as a function of depth, when the velocity search range used was: (a) $1 * v_{\max }$, and (b) $2 * v_{\max }$. The bottom plot (c) show both the estimates inside and outside the vessel as a function of depth. Estimation performed on the simulated data with a SNR of $20 \mathrm{~dB}$. Data are from the systole of the cardiac cycle.

[22], [27], and the velocity is close to zero. Thereby, the CMLE is capable of estimating even very small velocity levels. Again, this is a result of the similarity investigation, which captures the correlation properties among the velocities in a neighborhood and uses this in the estimation. This is beneficial in the subsequent processing, where it is determined which estimates should be superimposed on the gray-tone B-mode image.

\section{Performance on In Vivo Data}

The investigations on the simulated data reveal that the incorporation of the correlation property in the estimation is beneficial in both the estimation of the blood and the tissue velocities. A verification hereof on in vivo data has been carried out. Fig. 9 shows a typical example of the estimated velocity profiles for a healthy carotid artery with the CMLE (top) and MLE (bottom). As specified in Section IV, the temporal and spatial resolution is different from the simulated data, and the parameters $\Gamma_{1}$ and $\Gamma_{2}$ both take the value 100 in this case. Only the velocities inside the vessel are plotted, but the CMLE still outperforms the MLE by being able to produce almost nonzero estimates more often in the area outside the vessel. The advantage of the correlation property in the blood velocity estimation is demonstrated by the elimination of the outliers in the velocity profile. For the in vivo data one should be aware that the emitted pulse is narrow band. Usually estimators based on cross-correlation perform best on wideband pulses, but the results show that this is not a limitation with the CMLE in the current situation. 


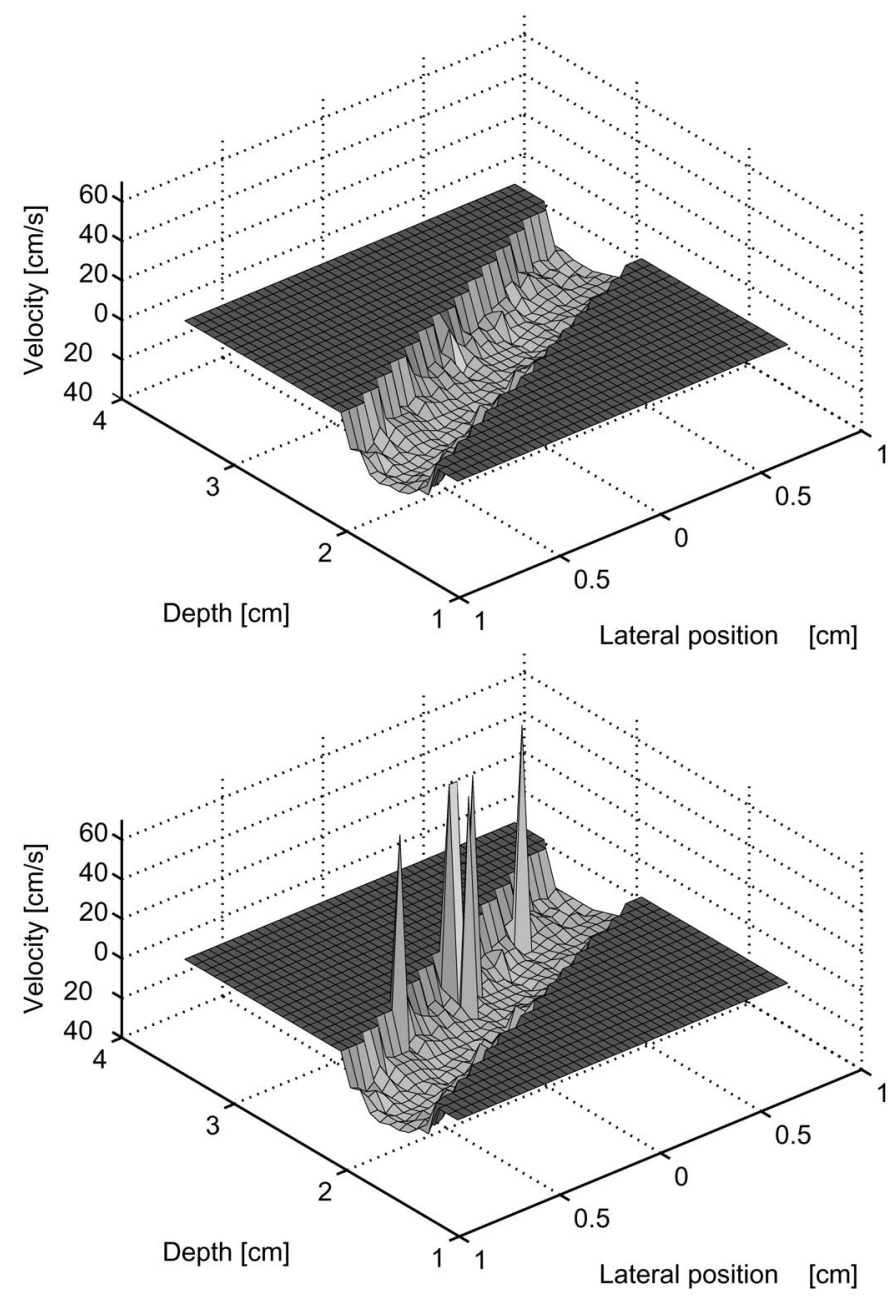

Fig. 9. Velocity estimates on in vivo data from the carotid artery with the CMLE (top) and MLE (bottom).

\section{Discussion}

In the derivation of the new maximum likelihood estimator, only non-turbulent flow conditions have been considered. Of course, this is a limitation, as turbulence and flow jets are seen in the heart at bifurcations and at the edges of a stenosis. This problem is encountered with most estimators as the emitted pulse interacts with scatterers at a range of velocities, and averaging over several samples are performed in the estimation. Still the aspect should be considered, and through simulations the capabilities of the estimator under these conditions must be determined. Part of this investigation involves a study of the best choice of number of correlation terms, $D_{j}$, and the values of the scaling parameters.

The blood flow profiles in the cardiovascular system differ for the different arteries and veins. For the femoral artery, both positive and negative velocities are present throughout the cardiac cycle. This will decrease the correlation between estimates in successive frames, and the level of correlation will depend on frame rate. These issues should be addressed through incorporation of information on frame rate, heart beat frequency, and similar variables in the computation of the scaling parameters multiplied onto the terms $D_{j}(j=1,2, \ldots)$. Increased frame rate is desirable as the velocity changes between frames will become smaller, the correlation between velocities will increase, and it will be easier to track the changes in velocities and use them in the estimator. New techniques to increase frame rate in color flow imaging [29] would be interesting to combine with this new estimator.

In the current study, the new maximum likelihood estimator was compared to two estimators also based on a cross-correlation analysis of the RF-data. Most commercial ultrasound scanners use the autocorrelation estimator, as it is computationally simple. The autocorrelation estimator, unfortunately, has an upper bound on the maximum velocity detectable, which is inversely proportional to the time between successively transmitted pulses along the same image direction. An increase in the maximum detectable velocity is at the expense of an undesirable lowering of the frame rate. This has been the impetus of the research into cross-correlation based estimators as discussed in Section I. A performance comparison of the autocorrelation estimator (AE) and the new estimator shows that the RMS will be slightly lower with the AE. Unfortunately, the detectable velocity range is lowered and the low amplitude tissue motion is not detected. The two latter features of the CMLE is desirable and encourage further research into use of the correlation property among the blood velocities in an estimator. With the advances within electronics, the increased number of computations required with the CMLE are of less concern.

\section{Conclusions}

A new estimator, which incorporates the features of fluid flow, has been derived and evaluated in this study. The aspect of correlation between blood velocities in time and space has been investigated, quantified, and incorporated. The new estimator (CMLE) is based on maximum likelihood theory. In its current form the CMLE uses the acquired RF-signals on complex form in the estimator. A performance evaluation on simulated and in vivo data from the carotid artery showed that the estimator is capable of performing blood velocity estimation and used the correlation property inherited from flow physics to improve on the estimation. For the current data sets the RMS errors were on the same order for the CC, MLE, and the CMLE estimators; but the use of a cross-correlation analysis alone puts an upper bound on the maximum detectable velocity with the CC and the MLE. This problem is circumvented with the CMLE; additionally, the estimator is capable of determining tissue motion at low velocities. The latter improves the basis for deciding which estimates to overlay onto the B-mode image. Thereby, the new estimator represents a promising estimator for blood velocity estimation in medical ultrasound imaging. In future investigations into its usability under turbulent flow conditions must be performed along with investigations of the influence of the settings of the spatial and temporal resolution. 


\section{ACKNOWLEDGMENT}

Morten Høgholm Pedersen, M.D., is thanked for helping out with acquiring the in vivo data. Thanassis Misaridis, Ph.D., Svetoslav Nikolov, Ph.D., Borislav Tomov, Ph.D., and Peter Munk, Ph.D., are acknowledged for their intensive work on building the dedicated sampling system used to acquire the in vivo data. We thank the reviewers for their valuable comments.

\section{REFERENCES}

[1] J. A. Jensen, Estimation of Blood Velocities Using Ultrasound: A Signal Processing Approach. New York: Cambridge Univ. Press, 1996.

[2] I. A. Hein and W. D. O'Brien, "Current time-domain methods for assessing tissue motion by analysis from reflected ultrasound echoes-A review," IEEE Trans. Ultrason., Ferroelect., Freq. Contr., vol. 40, pp. 84-102, 1993.

[3] K. Namekawa, C. Kasai, M. Tsukamoto, and A. Koyano, "Realtime blood-flow imaging system utilizing autocorrelation techniques," in Ultrasound '82. R. A. Lerski and P. Morley, Eds. New York: Pergamon, 1982, pp. 203-208.

[4] C. Kasai, K. Namekawa, A. Koyano, and R. Omoto, "Real-time two-dimensional blood flow imaging using an autocorrelation technique," IEEE Trans. Sonics Ultrason., vol. 32, pp. 458-463, 1985.

[5] T. Loupas, J. T. Powers, and R. W. Gill, "An axial velocity estimator for ultrasound blood flow imaging, based on a full evaluation of the Doppler equation by means of a two-dimensional autocorrelation approach," IEEE Trans. Ultrason., Ferroelect., Freq. Contr., vol. 42, pp. 672-688, 1995.

[6] T. Loupas, R. B. Peterson, and R. W. Gill, "Experimental evaluation of velocity and power estimation for blood flow imaging, by means of a two-dimensional autocorrelation approach," IEEE Trans. Ultrason., Ferroelect., Freq. Contr., vol. 42, pp. 689-699, 1995.

[7] D. Dotti, E. Gatti, V. Svelto, A. Uggè, and P. Vidali, "Blood flow measurements by ultrasound correlation techniques," Energia Nucleare, vol. 23, pp. 571-575, 1976.

[8] S. G. Foster, "A pulsed ultrasonic flowmeter employing time domain methods," Ph.D. dissertation, Dept. Elec. Eng., University of Illinois, Urbana, Ill., 1985.

[9] O. Bonnefous and P. Pesqué, "Time domain formulation of pulse-Doppler ultrasound and blood velocity estimation by cross correlation," Ultrason. Imag., vol. 8, pp. 73-85, 1986.

[10] K. W. Ferrara and V. R. Algazi, "A new wideband spread target maximum likelihood estimator for blood velocity estimationPart I: Theory," IEEE Trans. Ultrason., Ferroelect., Freq. Contr., vol. 38, pp. 1-16, 1991.

[11] K. W. Ferrara and V. R. Algazi, "A new wideband spread target maximum likelihood estimator for blood velocity estimationPart II: Evaluation of estimators with experimental data," IEEE Trans. Ultrason., Ferroelect., Freq. Contr., vol. 38, pp. 17-26, 1991.

[12] K. J. Parker and S. K. Alam, "System for estimating target velocity from pulse echoes in response to their correspondance with predetermined delay trajectories corresponding to different distinct velocities," U.S. Patent 5,419,331, priority date, 1994; granted 1995.

[13] S. A. McAleavey and K. J. Parker, "Effect of decorrelation on butterfly search velocity estimator performance," Proc. SPIEMed. Imag., vol. 4325, pp. 293-304, 2001.

[14] S. G. Foster, P. M. Embree, and W. D. O'Brien, "Flow velocity profile via time-domain correlation: Error analysis and computer simulation," IEEE Trans. Ultrason., Ferroelect., Freq. Contr., vol. 37, pp. 164-175, 1990.

[15] J. A. Jensen, "Range/velocity limitations for time-domain blood velocity estimation," Ultrasound Med. Biol., vol. 19, pp. 741$749,1993$.
[16] J. A. Jensen, "Artifacts in velocity estimation using ultrasound and cross-correlation," Med. Biol. Eng. Comp., vol. 32/4, Suppl., pp. 165-170, 1994.

[17] F. Forsberg, H. Oung, and L. Needleman, "Doppler spectral estimation using time-frequency distributions," IEEE Trans. Ultrason., Ferroelect., Freq. Contr., vol. 46, pp. 595-608, 1999.

[18] R. W. Fox and A. T. McDonald, Introduction to Fluid Mechanics. New York: Wiley, 1985.

[19] W. W. Nichols and M. F. O'Rourke, McDonald's Blood Flow in Arteries, Theoretical, Experimental and Clinical Principles. Philadelphia: Lea \& Febiger, 1990.

[20] H. L. Van Trees, Detection, Estimation and Modulation Theory, Part I: Detection, Estimation, and Linear Modulation Theory. New York: Wiley, 1971.

[21] H. L. Van Trees, Detection, Estimation and Modulation Theory, Part III: Radar-Sonar Signal Processing and Gaussian Signals in Noise. New York: Wiley, 1971.

[22] M. Schlaikjer, "Development and characterization of algorithms for estimation of blood velocity with ultrasound," Ph.D. dissertation, Ørsted•DTU, Technical University of Denmark, Kgs. Lyngby, Denmark, 2001.

[23] K. W. Ferrara and V. R. Algazi, "A statistical analysis of the received signal from blood during laminar flow," IEEE Trans. Ultrason., Ferroelect., Freq. Contr., vol. 41, pp. 185-198, 1994.

[24] K. W. Ferrara and V. R. Algazi, "A theoretical and experimental analysis of the received signal from disturbed blood flow," IEEE Trans. Ultrason., Ferroelect., Freq. Contr., vol. 41, pp. 172-184, 1994.

[25] J. A. Jensen, "Field: A program for simulating ultrasound systems," Med. Biol. Eng. Comp., 10th Nordic-Baltic Conf. Biomed. Imag., vol. 4, Suppl. 1, pt. 1, pp. 351-353, 1996.

[26] J. A. Jensen and N. B. Svendsen, "Calculation of pressure fields from arbitrarily shaped, apodized, and excited ultrasound transducers," IEEE Trans. Ultrason., Ferroelect., Freq. Contr., vol. 39, pp. 262-267, 1992.

[27] M. Schlaikjer, S. Torp-Pedersen, J. A. Jensen, and P. F. Stetson, "Tissue motion in blood velocity estimation and its simulation," in Proc. IEEE Ultrason. Symp., 1998, pp. 1495-1499.

[28] J. A. Jensen, O. Holm, L. J. Jensen, H. Bendsen, H. M. Pedersen, K. Salomonsen, J. Hansen, and S. Nikolov, "Experimental ultrasound system for real-time synthetic imaging," in Proc. IEEE Ultrason. Symp., 1999, pp. 1595-1599.

[29] S. I. Nikolov and J. A. Jensen, "In-vivo synthetic aperture flow imaging in medical ultrasound," IEEE Trans. Ultrason., Ferroelect., Freq. Contr., vol. 50, no. 7, pp. 848-856, 2003.

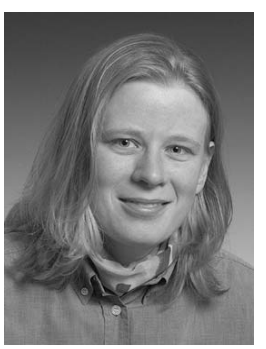

Malene Schlaikjer earned her Master of Science in electrical engineering in 1997 and the Ph.D. degree in 2001 from the Technical University of Denmark, Lyngby, Denmark. She currently holds a position as an assistent research professor at the department Ørsted $\bullet$ DTU at the Technical University of Denmark. Her main research interests are within blood velocity estimation with ultrasound and harmonic imaging.

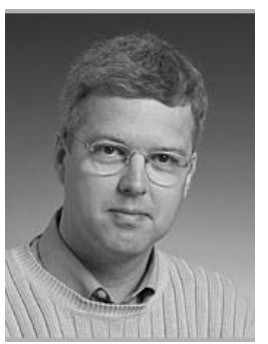

Jørgen Arendt Jensen (M'93-S'02) earned his Master of Science in electrical engineering in 1985 and the Ph.D. degree in 1989, both from the Technical University of Denmark, Lyngby, Denmark. He received the Dr.Techn. degree from the university in 1996. He has published a number of papers on signal processing and medical ultrasound and the book Estimation of Blood Velocities Using Ultrasound, Cambridge University Press in 1996. He has been a visiting scientist at Duke University, Stanford University, and the University of Illinois at Urbana-Champaign. He is currently full professor of Biomedical Signal Processing at the Technical University of Denmark at the Department Ørsted•DTU and head of Center for Fast 
Ultrasound Imaging. He has given courses on blood velocity estimation at both Duke University and University of Illinois and teaches biomedical signal processing and medical imaging at the Technical University of Denmark. He is one of the founders of a new biomedical engineering education jointly offered by Copenhagen University and the Technical University of Denmark. 